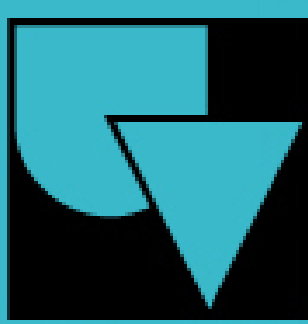

Universidad

del Valle

\title{
sociedad y economía
}

\section{Facultad de Ciencias Sociales y Económicas}

\section{Artículos}

Determinantes de la reintegración económica en excombatientes del departamento de Bolívar, Colombia

Jorge Luna-Amador • Amalfi Padilla-Castilla • Andrés Escobar-Espinoza

Pautas normativas y formas de racionalidad en la movilidad social afrodescendiente en Tuluá, Colombia

Juan Carlos Zuluaga-Díaz • Jefferson Jaramillo-Marín

Moda sostenible: más allá del prejuicio científico, un campo de investigación de prácticas sociales

Ana Martínez-Barreiro

Pobreza e indigencia en Argentina: construcción de una serie completa y metodológicamente homogénea

Guido Zack • Daniel Schteingart • Federico Favata

El concepto de vulnerabilidad macroeconómica. Una revisión de la literatura

Luis Hernando Portillo-Riascos

De la reactivación a la crisis: la industria argentina durante el ciclo de gobiernos kirchneristas

Pablo Manzanelli $\bullet$ Daniela Calvo

Entre asilos y hospitales psiquiátricos. Una reflexión historiográfica sobre el programa institucional de atención a la locura en Colombia María del Carmen Castrillón-Valderrutén

Humedal El Cortijo: Evidencia sobre la acción colectiva en defensa del ambiente en Santiago de Cali

Angélica Hernández-Morales • Angie Karina Rengifo-Fernández • Juan Pablo Jiménez-Herrera • Leydy Johana Narvaez-Ovalle 


\section{Moda sostenible: más allá del prejuicio científico, un campo de investigación de prácticas sociales}

Sustainable fashion: beyond scientific prejudice, a field of social practice research

Ana Martínez-Barreiro

Universidad de la Coruña, Coruña, España

ana.mbarreiro@udc.es

https://orcid.org/0000-0003-0385-0759

Recibido: 21-05-19

Aprobado: 12-02-20

1 Doctora en sociología. 


\section{Resumen}

En los últimos años se han producidos numerosas investigaciones dedicadas al estudio académico de la moda sostenible lo cual nos confirma que se están producido un conjunto de conocimientos y actitudes compartidas hacia el tema de la sostenibilidad. La principal contribución de este trabajo está en ofrecer una visión más integrada de la moda y la sostenibilidad que deriva de los trabajos más recientes de la moda sostenible; y también de la teoría del actor- red (ANT) y la teoría de las prácticas sociales (TPS).

Palabras clave: moda sostenible; sistema de moda; Teoría del Actor-Red; Teoría de las Prácticas Sociales; cultura material.

\section{Abstract}

In recent years there has been a great deal of research devoted to the academic study of sustainable fashion, which confirms to us that there is a shared body of knowledge and attitudes towards the issue of sustainability. The main contribution of this work is to offer a more integrated vision of fashion and sustainability that derives from the most recent works of sustainable fashion; and also from the theory of the actor-network (TAN) and the theory of social practices (TSP).

Keywords: sustainable fashion; fashion system; Actor-Network Theory; Theory of Social Practice; material culture. 


\section{Introducción}

Inicialmente, el estudio de la moda ha sido considerado un tema frívolo por la parte más seria de la investigación social. Esta creencia ha hecho que, durante algún tiempo, los que escribían sobre la moda lo hacían con discreción, ante el temor de ser acusados de frivolidad científica. Sin embargo, este prejuicio científico ha cambiado radicalmente, no solo porque es un sector económico importante a nivel mundial, sino porque es un sector con una gran transcendencia ambiental y sociocultural, además de una de las industrias más globalizadas (Gardetti, 2018). La moda es, por derecho propio, toda una institución que ocupa una posición estratégica en la presente postmodernidad (Lipovetsky, 1990). Vale, como muestra, la posición ideológica representada por la escuela francesa e integrada por Bourdieu (1991) y Baudrillard (1974), donde uno de los conceptos básicos es el de la diferenciación. Posteriormente, han surgido posturas más complejas producto del individualismo y la globalización que caracterizan a la nueva sociedad de consumo de masas. Autores centrados en la postmodernidad, como Lipovetsky (1990) y Maffesoli (1990), han investigado el papel de la moda de forma diferente, ya que en ellos no solo tiene cabida el consumo jerárquico de la moda, sino que este ha sido sustituido por el placer y por la comodidad: pasando del estatus-símbolo al estilo-símbolo (Martínez-Barreiro, 1998).

Las investigaciones en torno a la moda no han parado de crecer, en los últimos años, hasta formar el campo de estudios de moda (Rocamora y Smelik, 2016; Black et al., 2014, y Mora, Rocamora y Volonté, 2014a); y los estudios culturales han tenido un papel muy relevante en su desarrollo social, cultural, económico, al incorporar diversas disciplinas, como historia (Breward, 2003), sociología (Entwistle, 2000; Kawamura, 2005; Crane, 2000), psicología social (Tseëlon, 1995), antropología (Woodward, 2007), los estudios de la mujer (Paulicelli y Wissinger, 2013). Todos estos enfoques y otros muchos, que se encuentran en la unión de dos o más de las disciplinas mencionadas, han conseguido establecer una variedad de teorías y puntos de vista, desde la materialidad de la moda, la globalización, el consumo y la producción, los estudios postcoloniales, los estudios de género y los medios de comunicación. Sin embargo, los significados y las prácticas sociales, en torno a la sostenibilidad, todavía están por ser examinadas en su naturaleza interdisciplinar.

El objetivo principal de este trabajo es analizar los distintos significados de la moda sostenible y contextualizar los principales enfoques que han surgido, en Europa y Estados Unidos, en los últimos años. Las numerosas investigaciones sobre la moda sostenible confirman que se están produciendo un conjunto de conocimientos y aptitudes compartidas hacia el tema de la sostenibilidad en el ámbito de la moda. Para alcanzar dicho objetivo, en primer lugar, vamos a indagar ¿cuándo los estudios de moda muestran más interés por el tema de la sostenibilidad? En segundo lugar, vamos a introducirnos en el concepto de sostenibilidad, y esbozar algunas dimensiones analíticas de la sostenibilidad que necesitan una atención especial. En tercer lugar, conscientes de la naturaleza interdisciplinar de la moda sostenible, hemos de identificar los principales trabajos que relacionan el concepto de sostenibilidad con la industria de la moda y el consumo. Finalmente, a medida que la literatura sobre la moda sostenible se vuelve más rica, se necesitan discusiones analíticas que nos lleven a un diálogo más crítico de los principales enfoques y metodologías interpretativas. Por ello, abordaremos la Teoría del Actor- Red (ANT) y la Teoría de las Prácticas Sociales (TPS); ambos enfoques nos pueden ofrecer algunas vías fructíferas sobre la relación de la moda y la sostenibilidad.

Este trabajo sugiere poner la atención en la noción de moda como un sistema y no como un discurso estético (Lury, 2011) o de desigualdad social (Bourdieu, 1991). Los estudios de moda subrayan que el sistema de la moda se refiere a una industria en particular, a un sistema único y especial para la producción y el consumo de ropa que nació de los desarrollos históricos, sociales, culturales y tecnológicos en Europa (Lipovestky, 1990; Martínez-Barreiro, 1998). La visión de la moda como sistema ha sido abordada por Entwistle (2000) y, más recientemente, por la socióloga Kawamura (2005), al definirla como un "sistema de instituciones, organizaciones, grupos, 
productores, consumidores, eventos y prácticas, todos los cuales contribuyen a la creación de la moda, que es diferente al concepto de ropa o vestimenta" (p. 45). Mientras el concepto de ropa o vestimenta es la parte más material de la moda (Woodward, 2007), por el contrario, el concepto de moda se caracteriza, más que nada, por su dimensión sociocultural, además de su dimensión económica (Kawamura, 2005). En su explicación sobre el concepto de moda, Joanne Entwistle (2000) la definió como un "sujeto híbrido" que requiere el estudio de la interconexión entre la producción y el consumo; y cualquier análisis de la misma debe tener en cuenta varios agentes, instituciones y prácticas que formen intersecciones para producirla (Entwistle, 2000, p. 101).

En cuanto a la metodología, este trabajo se fundamenta en el desarrollo teórico y contextual de las cuestiones planteadas anteriormente. Sin olvidar que indagar en la moda sostenible significa pensar en una amplia gama de teorías y conceptos, que pueden ayudar a comprender la relevancia de la moda sostenible desde la teoría social y cultural, además de la cultura material de la ropa o la vestimenta (Bovone, 2015). Entre las conclusiones observadas, debemos enfatizar que la sostenibilidad se ha convertido en una categoría esencial para el estudio académico de la moda en el siglo XXI. Además de señalar la dificultad de encontrar una perspectiva unitaria, capaz de abordar la complejidad ambiental, social y cultural de los fenómenos relacionados con el sistema de la "moda sostenible". Finalmente, varios investigadores que han abordado este tema, desde diferentes perspectivas disciplinarias, coinciden en señalar que la mayor dificultad para el desarrollo de la moda sostenible es la constitución de un nuevo imaginario de la moda más inclusivo, plural y sostenible (Tseëlon, 2011; Carfagna et al., 2014).

\section{La sostenibilidad en los estudios de la moda}

A pesar del prejuicio científico inicial, la investigación de la moda ha tenido una larga historia en la sociología clásica (Simmel, 1957; Veblen, 1974) como también emplazó el interés de la sociología moderna (Lipovetsky, 1990;
Bourdieu, 1991; Entwistle, 2000; Kawamura, 2005; Crane, 2000; Martínez-Barreiro, 1998). Sin embargo, no existieron muchos trabajos sobre la moda y la sostenibilidad, exceptuando los estudios sobre la responsabilidad social de las empresas, a través del enfoque de las $3 \mathrm{P}$ o del Triple Bottom Line (TBL), de Elkington (1998). La explicación de por qué el concepto de sostenibilidad llamó poco la atención de la sociología de la moda -y que surgiera tarde- está en la propia división sutilmente trazada entre el mundo natural y el mundo social (Entwistle, 2015). Los actuales costos ambientales ejemplifican esta división de la "naturaleza" y el "mundo social", cuyo resultado ha sido insuficientemente analizado por la sociología de la moda, al centrar su interés en las desigualdades sociales, o en los estudios culturales y las propias prácticas de consumo de ropa (Entwistle, 2015). Sin embargo, en los últimos años, el tema de la sostenibilidad ha estado a la vanguardia de algunas de las reflexiones más estimulantes sobre la moda (Mora, Rocamora y Volonté, 2014b). Los primeros debates sobre la cuestión de la sostenibilidad se remontan a mediados de la década de 1990, cuando el tema de la explotación infantil -por parte de algunos proveedores de la marca Nikéllamó la atención de la sociedad occidental (Mora et al., 2014b). A partir de ese momento, distintas organizaciones no gubernamentales, como Greenpeace y Clean Clothes Compaign instaron a las marcas de moda a avanzar hacia modelos y prácticas comerciales más sostenibles. Sin embargo, para que se estableciera el tema de la sostenibilidad en los estudios académicos de la moda tuvimos que esperar a que se publicaran algunos trabajos, como por ejemplo la segunda edición de la obra The Fashion Reader, escrita por Welters y Lillethun (2011). Obra donde se observa, por primera vez, un cambio en el análisis temático de la investigación sociológica de la moda y en las metodologías utilizadas para su estudio. Presenta una información muy detallada sobre los impactos sociales, económicos y ambientales que el sector textil y de la moda produce. Al mismo tiempo, explica las posibles prácticas alternativas para articular la sostenibilidad en el diseño y la propia innovación social. 
En esta última línea de investigación, varios trabajos han conseguido desafiar las ideas preexistentes de la sostenibilidad en la industria textil y de la moda, como Kate Fletcher (2008), que en su libro Sustainable Fashion and Textiles, propuso un enfoque holístico del tema de la sostenibilidad en el sector de la moda. Bajo el supuesto de que diseñar un producto significa tener en cuenta todo el ciclo de vida del producto, desde la obtención de los materiales hasta los propios procedimientos de producción y el diseño, las prácticas de consumo y la eliminación de las prendas que sigue al uso y al consumo de ropa. Igualmente, en esta misma época, Sandy Black (2008) comienza a discutir los parámetros históricos y culturales relacionados con el surgimiento de una nueva estética de la moda: el lujo sostenible. Tendencia que promueve la "ecología" en las nuevas colecciones de moda. Igualmente, Sandy Black (2008), en su libro Eco-Chic: The Fashion Paradox, revela que -a principios del siglo XXI, en Gran Bretaña-algunas marcas de prestigio comienzan a considerar la sostenibilidad ambiental y social, tanto en sus colecciones como en la comunicación con los clientes. En ese mismo período, la sociología italiana cuestiona la Responsabilidad Social de las Empresas (RSC), por su falta de atención a los valores éticos, e introduce nuevos conceptos como el de "la moda orgánica" y "el consumo responsable" (Lunghi y Montagnini, 2007; Bovone y Mora, 2007). Posteriormente, tras la aparición de un consumidor más consciente, algunas empresas comienzan a redefinir sus reglas y valores básicos, emergiendo incluso el comercio justo (Rinaldi y Testa, 2014). Todos estos cambios se hacen más visibles gracias a la publicación The Handbook of Fashion Studies, escrito por Sandy Black et al. (2014). A partir de ese momento, el tema de la "sostenibilidad" comienza a formar parte de los estudios de moda, tras la publicación de un capítulo que se titula: $\mathrm{La}$ moda sostenible en un mundo globalizado. En este se investiga cuáles son las relaciones entre la sostenibilidad y la moda rápida, la globalización y la moda, los derechos humanos, las pequeñas y las grandes empresas, y las estrategias de diseño sostenible. Hasta ese momento, la teoría de la moda no se había interesado ni preocupado por el impacto ecológico y social de la moda; y hubo que esperar al inicio del siglo XXI para que el sistema de la moda adoptara la terminología de la sostenibilidad.

A partir de ese momento, el interés por la sostenibilidad ha aumentado de manera progresiva, consolidándose una nueva perspectiva de investigación dentro de los estudios de moda, y donde las redes de referencias se han vuelto más extensas. Por ejemplo, varias revistas científicas -The Fashion Theory, International Journal of Fashion Studies, The Journal of Dress and Body y Economy and Society- han encaminado todos sus esfuerzos en promover el diálogo sobre la moda y la sostenibilidad. No obstante, su naturaleza interdisciplinaria requiere combinar diferentes disciplinas como la economía, la historia, la ciencia política, la sociología, la antropología, el derecho y la biología; además de emplear diversas metodologías desde los estudios estadísticos, la modelización teórica, el análisis cualitativo o el estudio etnográfico (Kawamura, 2011). Incluso se han desarrollado proyectos donde se reconoce explícitamente la colaboración internacional. Por ejemplo, el International Trade Center (ITC) -un organismo conjunto de las Naciones Unidas y la Organización Mundial del Comercio, con sede en Ginebra (Suiza)- ha promovido muchos proyectos a nivel internacional, bajo la iniciativa de la moda ética. Esta iniciativa ha involucrado a empresas y diseñadores de moda (a menudo mujeres), en pequeñas comunidades africanas, con el objetivo de desarrollar habilidades artesanales locales y así promover la creatividad y la capacidad de diseño (Menkes, 2012). A nivel nacional, en los países nórdicos, Pedersen y Gwodzd (2014) han involucrado a las empresas y diseñadores en sus proyectos, con la finalidad de aumentar la conciencia ambiental y social de los consumidores.

\section{El universo significativo de la moda sostenible}

En este epígrafe vamos a introducirnos en el concepto de sostenibilidad y esbozar algunas dimensiones analíticas que en nuestra opinión necesitan una atención especial. El concepto se utilizó por primera vez en el Informe Bruntland 
-publicado por las Naciones Unidas con el título Nuestro futuro común, en el año 1987- donde sostenibilidad se define como "el desarrollo que satisface las necesidades del presente; sin comprometer las necesidades de las futuras generaciones" (WCED, 1987, p. 43). Una de las principales propuestas de la sostenibilidad está en observar cómo actúa la naturaleza y seguir los principios que la rigen, como cooperación, simbiosis y eficiencia (Salcedo, 2014, p. 14). Si bien el primer modelo propuesto para el desarrollo sostenible fue un intento de armonizar el desarrollo humano con los límites de la naturaleza, no obstante, el objetivo del desarrollo sustentable es tener un alcance global (Doppelt, 2012).

En la actualidad, la "sostenibilidad" tiene tres significados que van más allá de su asociación con el medio ambiente. El primer modelo afirma que "la sostenibilidad es mucho más que nuestra relación con el medio ambiente; se trata de nuestra relación con nosotros mismos, nuestras comunidades y nuestras instituciones" (Seidman, 2007, p. 58). El segundo modelo asocia y define la sostenibilidad como la armonía entre tres pilares: la economía, el medio ambiente y la igualdad social (Salcedo, 2014; Gardetti, 2018). El tercer modelo, constata que el concepto de "sostenibilidad" está muy relacionado con las organizaciones, y que, por lo tanto, ha de ser utilizada a nivel general de la sociedad. A su vez, Suzuki y Dressler (2002) especifican que la sostenibilidad también ha de ser entendida, a nivel individual, como la evaluación de todos los comportamientos humanos con la visión de reformular los comportamientos que contradicen el desarrollo de un futuro sustentable. Sin embargo, recientemente, Gardetti (2018) subraya que la "sostenibilidad" no es solo un nuevo concepto, sino también un nuevo paradigma. El término paradigma viene de la palabra griega "paradeima" que significa un nuevo modelo o patrón. Esto implica ver al "sistema de la moda" de una forma diferente a la que domina nuestro modelo actual; y empezar a pensar en la moda y la sostenibilidad de una forma más profunda, sólida e integrada, e integrar nuevos valores (Entwistle, 2015). Por ejemplo, Gardetti (2018), siguiendo a Hethorn y Ulasewicz (2008), especifica que la "moda sostenible simboliza que durante el desarrollo y uso de una prenda no se hizo daño a las personas ni al planeta, y que incluso puedan mejorar el bienestar de las gentes que interactúan con ellas y también en el ambiente en que se desarrollaron y son usadas" (Gardetti, 2018, p. 35).

Llegados a este punto, hemos de analizar ¿qué es la moda sostenible? La terminología utilizada en el discurso académico de la moda ha evolucionado de "moda ecológica" hacia un mayor uso del concepto "moda sostenible". Este último concepto es más inclusivo, al englobar temas como la justicia, la equidad, la economía, etc. Este léxico ha sido discutido por distintos expertos, explorando los cuatro términos de manera diferente. 1) Eco-Moda representa a las prendas de vestir producidas con métodos muy poco perjudiciales para el medio ambiente; y de hecho se asocia con los materiales ecológicos y las certificaciones ecológicas (Henninger, Alevizou y Oates, 2016). 2) Moda Ética enfatiza el aspecto medio ambiental y el social de la moda, al asociarse más con las condiciones de trabajo más justas y los modelos de negocios más sostenibles (Bovone, 2015; Crane, 2010; Salcedo, 2014). 3) Slow Fashion surge, tal como afirma Kate Fletcher (2010), como un movimiento social en respuesta a los ciclos rápidos de la moda; por ende, este concepto no solo se basa en el tiempo, sino también en la calidad (Clark, 2008). Y donde el consumidor tiene un papel central.4) La Moda Sostenible se caracteriza por incluir todos los enfoques anteriores (desde la promoción de unas buenas prácticas sociales y medio ambientales), incluyendo la reducción de la producción y el consumo de ropa, hasta promover el reciclaje y el uso de materiales renovables y orgánicos. Fletcher y Grose (2012), y Henninger et al. (2016) subrayan que la moda sostenible significa alejarse de las prácticas de producción y consumo del sistema de la moda rápida.

\section{Enfoques teóricos de la moda sostenible}

En los últimos años, se han producido numerosas investigaciones en Europa y Estados Unidos dedicadas al estudio de la moda y la sostenibilidad, lo cual nos confirma que está creciendo 
un conjunto de conocimientos y actitudes compartidas hacia el tema de la moda sostenible (Mora et al., 2014b). Su naturaleza interdisciplinaria nos ha llevado a la siguiente pregunta: ¿cuáles son los principales enfoques teóricos de la moda sostenible? La moda sostenible abarca no solo tres enfoques: el ecológico, el social y el económico, sino que, recientemente, se ha incorporado otro enfoque: la educación para el desarrollo sostenible (EDS).

1) El enfoque ecológico de la moda está en línea con el movimiento Slow Fashion, fundado por Kate Fletcher en el año 2007. Este enfoque es inherente al estudio del ciclo de vida de las prendas de vestir y esta personificado por Fletcher (2008), Slater (2003), Gwilt y Rissanen (2011), Salcedo (2014), y otros muchos académicos que han abordado este tema desde diferentes perspectivas y en distintos ámbitos. Para valorar los impactos de un producto, a lo largo de su ciclo de vida, se ha desarrollado el "Análisis del Ciclo de Vida" (ACV) de un producto. Método que analiza los impactos ambientales que ocurren desde que se extraen las materias primas, pasando por la producción, hasta el uso por parte del consumidor y el mismo desecho del producto (Salcedo, 2014, p. 19). En esta línea, Gwilt y Rissanen (2011) han adoptado un enfoque muy práctico respecto a la configuración de la sostenibilidad en el sistema de la moda, dando ejemplos sobre los diversos métodos de diseño, desde cero residuos en el corte, hasta la reutilización de materiales de desecho, y así ayudar al desarrollo de nuevas estrategias de diseño sostenible. Igualmente, Gwilt (2014) elaboró un manual académico con una información muy accesible sobre la práctica de la moda y el diseño sostenible para los estudiantes de las escuelas de diseño de moda. Dentro de la perspectiva del ciclo de vida (ACV) existen muchos trabajos, desde el estudio del impacto de los materiales que la industria textil genera sobre el medio ambiente (Van der Velden, Patel y Vogtländer, 2014), hasta el estudio de la huella de carbono que produce la moda rápida (Choi, 2013). Además de un análisis comparativo de las políticas sostenibles en distintas empresas (Caniato, Caridi, Crippa y Moretto, 2012). Incluso el uso de las etiquetas y certificaciones en la ropa del sector de la moda (Jucker, 2012). Todos estos estudios revelan un gran interés por adquirir nuevos conocimientos sobre cualquier etapa de la cadena de valor.

2) El enfoque de la justicia socialy las cuestiones relativas a los derechos de los trabajadores es inseparable del impacto de la globalización económica, que facilitó la flexibilidad del sistema de la moda, gracias a la utilización generalizada del sistema de subcontratación, en los países en vías de desarrollo, produciendo efectos sociales indeseables (Harris, 2017; Bradley y Healy, 2008). Pues la deslocalización de la producción textil ha empeorado las condiciones laborales, con bajos salarios y las largas jornadas de trabajo (Ross, 1997, p. 11). Para algunos investigadores, la desigualdad de género está muy vinculada con la insostenibilidad de la moda (Phizacklea, 1990; Raghuram, 2004). Por ejemplo, en los últimos años ha florecido una abundante literatura que insiste en denunciar estas prácticas de explotación laboral, como la publicación No Logo de Klein (2001) y también en otros trabajos publicados una década más tarde, como los de Siegle (2011) y Cline (2014). Investigaciones que documentaron las prácticas de trabajo esclavo detrás de varias marcas de moda, hasta el punto en que los bajos costes del empleo pueden no compensar la caída de la facturación derivada, por boicot a una marca. Lohmeyer y Schüßler (2017) señalan que dicho problema no puede ser solo resuelto por parte de las empresas a través de los códigos de conducta, sino que inclusive es muy importante la presión del consumidor (Warde, 2005). Dentro de este enfoque, se insiste en la posibilidad de que la producción de moda también pueda servir para fomentar la sostenibilidad social, mediante el aprovechamiento de un vínculo especial que conecte la creatividad y la inclusión social (Santagata, 2010). Esta idea tiene sus raíces en la teoría sociológica de Richard Sennett (2008), al argumentar que una buena artesanía implica una buena ciudadanía, donde el ritmo y el aprendizaje de un trabajo creativo enseñan a gobernarse a sí mismos y ser buenos "ciudadanos". Asimismo, Lunghi (2012) también ha abordado el papel desempeñado por la creatividad, en el arte, para apoyar la inclusión social de las personas con discapacidad. 
3) El enfoque del consumo sostenible es inherente al debate académico sobre cómo mitigar el cambio climático y reducir las emisiones de dióxido de carbono (Co2), a través de los hábitos de consumo (Warde y Southerton, 2012, p. 2). Si bien la publicación de Nuestro Futuro en Común (WCED, 1987) logró extender la expresión del desarrollo sostenible entre ecologistas y gobiernos. Sin embargo, la atención científica se ha centrado más en el "cambio de comportamiento" (Warde y Southerton, 2012, p. 1). La idea básica de este enfoque es que solo cuando los consumidores, en masa, cambien cómo viven sus vidas, se podría lograr un futuro más sostenible desde el punto de vista ambiental y social. Sin embargo, la forma en que se conceptualiza el comportamiento y cómo se produce el cambio está sujeta a una discusión teórica (Warde y Southerton, 2012). De hecho, Crane (2010), a la hora de conceptualizar el comportamiento del consumidor, observa una variedad de factores: subjetivos (identidad, cultura y estatus social); objetivos (los recursos, los conocimientos); donde el discurso político y moral les exhorta -o no- a participar en este tipo de consumos. Especifica que la forma de interpretar las interrelaciones entre estos factores y cómo los consumidores acuerdan los diferentes atributos, en su consumo, se encuentra en los conceptos de "habitus" y "estilo de vida" de Bourdieu (1991). Asimismo, observa que, en los estudios que han examinado las relaciones entre las actitudes hacia los tipos específicos de consumo más responsables, se encontró una discrepancia entre las actitudes y el propio comportamiento (Crane, 2010, p. 367). Por ejemplo, en el estudio realizado por el Eurobarómetro367, a los veintisiete países miembros de la Unión Europea, se encontró que "mientras que el $75 \%$ de los encuestados de la encuesta dice que están listos para comprar productos ecológicos si son más caros, solo el $17 \%$ lo ha hecho en realidad el mes anterior a la encuesta" (Comisión Europea, 2013, p. 55) Por otra parte, varios expertos consideran que, a medida que los consumidores se vuelven más "conscientes" del daño que la producción de prendas de vestir ocasiona, es posible afirmar que puedan ajustar los hábitos de compra o consumo a un mayor nivel de conciencia ambiental (Carfagna et al., 2014). Cabe subrayar que, en un estudio realizado a los consumidores de $\mathrm{H} \& \mathrm{M}$, se descubrió que muchos consumidores buscan minimizar los efectos ambientales negativos producidos por la industria de la moda rápida (Shen, 2014).

Dentro de esta última perspectiva, ya se están dando otras opciones de consumo, como el intercambio de ropa o swaps parties. Evento donde se intercambian prendas, que suelen estar en buen estado, para darles un segundo uso con las mismas garantías de un artículo recién comprado. Esta práctica de consumo tiene gran presencia en el mundo virtual: Swap Style ${ }^{2}$. Lo interesante de esta acción es que se ha convertido en una práctica responsable que evita tirar a la basura grandes cantidades de ropa. Otras prácticas que minimizan los impactos sobre los hábitos de consumo son la reutilización y aceptación de la ropa de segunda mano o moda vintage (Heike, 2015). Esta práctica es un claro indicador de la ampliación de la vida útil de las prendas, según lo investigado por la antropóloga Karen Tranberg Hansen (2000). Por último, otra práctica a la hora de afrontar el exceso de residuos es el "upcycled"; término acuñado por McDonough y Braungart (2002), al crear materiales valiosos como, por ejemplo, los polares de la marca Patagonia. Estos son fabricados a partir de botellas PET recicladas, gracias al programa de I+D del reciclaje textil. Según Tim Jackson (2011), el reciclaje es una estrategia de transición a la hora de minimizar los impactos y propone un cambio de modelo: eliminar por completo los residuos. Planteando la teoría de cradle to cradle, o economía circular, donde los residuos se conciban como algo útil $\mathrm{y}$, por tanto, dejen de ser residuos y pasen a convertirse en materia prima para otros sistemas, ya sean tecnológicos o biológicos.

4) El discurso académico de la moda sostenible incluye la Educación para el Desarrollo Sostenible (EDS), lo cual supone no solo una reorientación de los sistemas educativos, sino preparar a los ciudadanos y a las administraciones con nuevos conocimientos, según la UNESCO (2005). El enfoque de la EDS está pensado para transformar el sector industrial actual y preparar a los

\footnotetext{
2 www.swapstyle.com
}

sociedad y economía • e-ISSN: 2389-9050 • No. 40, 2020 • pp. 51-68 
alumnos para liderar ese cambio. En este tema, Parker y Dickson (2009); MacVaugh y Norton (2012); y, recientemente, Gardetti y Delgado (2018) han reconocido que el sistema de la moda sostenible requiere un enfoque más integrado e interdisciplinario de los problemas ambientales, sociales y económicos, junto con un programa vital de educación para las personas que trabajan dentro del sistema de moda y los consumidores, en general. Igualmente, en este mismo enfoque, otros investigadores constatan la necesidad de prestar más atención a la dimensión cultural de la sostenibilidad. En la actualidad, se necesitan más estudios sobre la posible constitución de un imaginario de moda sostenible, ya que el campo de la moda promueve ideales altamente divisivos en términos de estética corporal, clase, raza o sexualidad. De ahí, la necesidad de plantear la siguiente pregunta ¿cómo puede construirse y mantenerse un imaginario que abarque la pluralidad, la tolerancia, la inclusión y el multiculturalismo? Los estudios sobre la moda modesta (Lewis, 2013) y de tallas grandes (Canina y Volonté, 2014) constituyen un impulso importante para llevar a la moda fuera de sus valores hegemónicos, sobre todo hacia un imaginario de moda más inclusivo y plural. De hecho, el trabajo de Tseëlon (2011) revela todas las contradicciones que se pueden observar entre el negocio de la moda y la búsqueda de la ética.

\section{La Teoría del Actor-Red (ANT)}

A medida que la literatura sobre la moda sostenible se vuelve más rica, se necesitan nuevos enfoques y metodologías interpretativas, que puedan respaldar el desarrollo de futuras investigaciones de la moda sostenible. Con este objetivo, vamos a considerar la Teoría del Actor-Red (ANT). La Teoría (ANT) se presenta como una propuesta metodológica radical y omnicomprensiva, planteando un desafío a las ideas habituales que podemos encontrar en la teoría sociológica de la moda (Entwistle, 2016). Uno de los aspectos centrales de esta teoría es que plantea la investigación de la moda como un "híbrido" entre la naturaleza y la cultura (Entwistle, 2015; 2016). Lo cual nos permite ver las continuidades entre las prácticas de vestir y su amplio impacto ambiental y, al mismo tiempo, nos da la oportunidad de empezar a pensar de forma diferente sobre cómo conectamos la naturaleza y la cultura. Y, de esta forma, comenzar a asignar, establecer, fijar o ensamblar las relaciones sociales y culturales entre nuestra ropa y el medio ambiente (Entwistle, 2016).

Para comprender el valor de este enfoque en los estudios de la moda, hemos de tener en cuenta los siguientes principios, y la propuesta metodológica en la que se fundamenta la Teoría del Actor-Red. En primer lugar, Bruno Latour (2007) ha conseguido desafiar algunas ideas preconcebidas sobre lo que es ser "moderno". Según él, la modernidad se contextualizó bajo la distinción artificial entre "naturaleza" y "cultura" y, a su vez, sostiene que el punto de vista de la "cultura" se ha visto como superior y activo; por el contrario, a la "naturaleza" se la vio como inferior y pasiva.

En segundo lugar, Latour (2008) subraya que los objetos que denominamos como "naturales" son en realidad productos históricosociales de nuestras formas particulares de ver el mundo; y son, por lo tanto, objetos o artefactos naturales. A partir de este supuesto, Latour (2007) considera que "los objetos" son simples híbridos de la relación naturalezacultura. Latour y Woolgar (1995) consideran que, de la misma forma, la ciencia moderna depende de instrumentos y dispositivos como los microscopios, que permiten ver bacterias o células invisibles a simple vista. Igualmente, Latour (2008) presupone que la naturaleza ya no puede ser vista como algo que esté "ahí fuera", o separada y diferente de nosotros, sino que hemos de vernos de forma continua con la naturaleza, como una parte de ella. Al efecto, nuestro parecer social basado en nuestra ropa y vestimenta es en realidad un "híbrido" de la relación entre la naturaleza y la cultura. El enfoque de Bruno Latour nos puede ayudar a abrir ese diálogo entre la ciencia y la cultura, entre la tecnología y la moda. La proximidad entre el saber formal de la ciencia y la tecnología con su dimensión humana ya se investiga en los estudios de Ciencia, Tecnología y Sociedad (CTS). 
En tercer lugar, si seguimos con esta lógica de ver la moda como un hibrido entre naturaleza y cultura, parece claro que algo tan aparentemente "cultural", como son las propias creaciones de la moda, sea una creación híbrida entre la naturaleza y la cultura. Las prendas de ropa son algo "natural", ya que están compuestas por materiales naturales, (como el algodón, la seda y el lino) y, a su vez, son "montadas y ensambladas" en nuestro mundo social y cultural de manera compleja, a través de las cadenas globales de producción textil (Gereffi y Korzeniewicz, 1994). Las cadenas globales de producción hacen hincapié en las conexiones dentro del sistema de producción, que se conoce como empresa red (Castells, 2000). El concepto de red ha tenido un desarrollo muy importante en el ámbito geográfico y relacional de la moda (Crewe, 2004). Por otra parte, está la Teoría del Sistema Mundo (TSM), que tiene su origen en la teoría marxista y cuyo objetivo es rastrear las conexiones entre "las regiones-periféricas" -en las que se encuentra la mano de obra barata- con el "núcleo" de las zonas occidentales más desarrolladas (Wallerstein, 1974; Gereffi y Korzeniewicz, 1994). Los trabajos de la Teoría del Sistema Mundo (TSM) nos pueden servir como una herramienta muy útil para el análisis de la insostenibilidad de la moda rápida y las dificultades de los países en desarrollo. Por consiguiente, en este nuevo contexto, ya no encajaría en absoluto hacer una clara distinción entre las dimensiones "naturales" y "culturales" de la moda, sino simplemente examinar el flujo continuo de materiales, objetos y actores que componen el vestido de moda. En consecuencia, tal como sugiere la teoría del Actor-Red (ANT), los mundos natural y social son el producto de redes de relaciones entre actores. Luego, en este nuevo escenario, debemos comenzar a pensar de una forma más ecológica, es decir, vernos como parte de la ecología de la tierra $\mathrm{y}$, de ninguna manera, separados o por encima de ella. Pensar en la moda como un híbrido entre naturaleza y cultura puede ser muy ventajoso en el estudio sobre ella.

En cuarto lugar, esta nueva forma de pensar depende de una comprensión diferente del significado de "actor", ya que en la teoría (ANT) los actores son tanto humanos como no humanos. En esta teoría, un "actor" o "actante" es cualquier cosa con la capacidad de actuar de alguna manera; por ejemplo, el agua es un actor o "actante", ya que es un componente activo de los materiales que componen la ropa. Igual que el algodón, la seda, la lana, el lino, etc. La teoría (ANT) es útil para ayudar a determinar cómo las categorías de "personas" y "cosas" están en juego e interactúan en la "moda". Ya que, por el principio de "simetría" de Callon (1986), ninguna de las dos tendrá prioridad, ni será vista como una entidad separada o superior. En este punto, la teoría (ANT) rechaza la división ontológica de la materialidad y el significado, y la prioridad del significado. En cambio, asume que los materiales y las cosas se consideran una parte fundamental de los ensamblajes que llegan a constituir lo social (Latour, 2008). Al utilizar este marco teórico en el estudio de la "moda", se abordaría como un conjunto de elementos humanos y no humanos, o "actantes" que componen el fenómeno de la moda en cuestión. Este enfoque todavía no está muy bien desarrollado dentro de los estudios de la moda. Sin embargo, Entwistle y Slater (2014) han conseguido investigar a las modelos de moda desde la perspectiva de la ANT. Este artículo no solo propone que debamos adoptar este nuevo enfoque, sino que debemos aprender a pensar sobre las nuevas facetas de la moda a través de sus múltiples elementos materiales y humanos, y sus relaciones.

En quinto lugar, esta teoría aporta una metodología para la "localización" de estas conexiones, y nos puede enseñar a observar y a seguir a los actores, con la finalidad de ver a dónde van y lo que hacen. De hecho, no es una teoría explicativa, sino descriptiva. Llegados a este punto, podemos atestiguar que, si utilizamos esta metodología sobre las prendas de moda, podríamos comenzar con los campos de algodón en la India, o las fábricas de producción en algún lugar de Asia. Y seguir a los actores desde la producción de textiles a su ensamblado textil, su distribución a las tiendas y llegar hasta los consumidores. Incluso ir más allá del consumo, llegar hasta la eliminación de los residuos de ropa. Esto significa que, si seguimos a las prendas de ropa, podemos observar cómo los actores o actantes "naturales" entran en contacto con los agentes "culturales"; y de esa forma se ensamblan en sistemas complejos o híbridos entre la naturaleza y la cultura. La Teoría del Actor- Red puede resultar muy útil para investigar la moda sostenible. 


\section{La Teoría de las Prácticas Sociales (TPS)}

El desarrollo de la moda sostenible requiere cambios transformadores en las prácticas de consumo de todos los involucrados. Por ello, debemos tener en cuenta el potencial explicativo de la Teoría de las Prácticas Sociales (TPS) y su transición hacia la sostenibilidad. Esta teoría ha adquirido cierta relevancia en las prácticas de comer (Evans, 2012; Warde, Cheng, Olsen y Southerton, 2007), y de higiene personal del cuerpo (Shove, 2003). Sin embargo, ha tenido un escaso desarrollo en relación con la ropa, excepto en algunos trabajos como los de Klepp y Bjerck (2014), Bellotti y Mora (2014), y Woodward (2007). El valor del enfoque de la TPS está en: a) la capacidad de repensar y diseñar acciones orientadas hacia la transición de nuevas formas de consumo sostenible; b) la capacidad para enfocarse en el consumo como el uso de cosas al servicio de la promulgación de prácticas sociales; c) poner su atención en la dinámica micro-sociológica sobre la cual se estructuran las dinámicas de las prácticas de consumo sostenible (Warde, 2005). En otras palabras, la TPS propone entender las distintas formas de consumo, como resultado de la dinámica de las prácticas que organizan la vida cotidiana de los usuarios.

Shove y Pantzar (2005) siguieron a Schatzki (1996), quien se propuso definir a las prácticas como una forma rutinaria de la conducta social compuesta por un "nexo de formas de decir y de hacer que tienen cierta dispersión espacial y temporal" (p. 89). Dentro de este mismo concepto, Shove, Pantzar y Watson (2012) distinguen tres componentes básicos de toda práctica. 1) El conocimiento práctico (el "saber hacer"). 2) Las materialidades (los objetos materiales y recursos que constituyen la práctica). 3) El sentido (el conjunto de significaciones y valoraciones que constituyen cada práctica). Esto significa que cada práctica depende, para su existencia, de la interrelación activa de estos tres elementos. Una de las fortalezas centrales de esta teoría es que la práctica, como unidad de análisis, no se refiere únicamente al ámbito de la intencionalidad individual, sino también a un conjunto de materialidades y significados que se movilizan en ella y anteceden a su ejecución individual. Por consiguiente, dentro de la vida social, las prácticas han de ser entendidas como un entramado, cuya dinámica da forma a la vida cotidiana, estructurando las prácticas de vestirse o asearse. En concreto, la teoría de las TPS, respecto a las prácticas sostenibles, tiene la fortaleza de a) visibilizar los aspectos más rutinarios y convencionales de la vida social; b) explicar los cambios en los patrones de consumo y éxito (o no) de la transición hacia los consumos más sostenibles (Shove, 2010); c) la TPS, al hacer de la práctica su principal unidad de análisis, propone una aproximación totalmente distinta, que desarrolla formas de explicación y de intervención en el área del comportamiento pro ambiental. Estas van más allá de lo puramente motivacional o de los estilos de vida.

Llegados a este punto, en el nuevo paradigma de la "moda sostenible", debemos comenzar a prestar una cuidadosa atención a las prácticas de consumo y al propio diseño de ropa. Por ejemplo, algunos autores ya han subrayado cómo el diseño de determinados objetos, que se utilizan en la vida cotidiana, puede mejorar la calidad de vida de las personas, como ocurrió en el caso del "diseño nordic walking" (Pantzar y Shove, 2005). Igualmente, Skjerven y Reitan (2017) han reconocido que la competencia en el diseño de ropa también se puede convertir en una herramienta útil para crear un entorno social, cultural y físico más sostenible. Y, desde el consumo, Klepp y Bjerck (2014) han conseguido investigar cómo las prácticas de usar y tirar la ropa pueden influir en el medio ambiente. Inclusive han abordado la pregunta ¿cómo cambiar las prácticas de lavandería? Pues el desarrollo de prácticas colaborativas, como el intercambio de lavadoras en edificios residenciales, han ayudado a la redefinición de los estándares culturales, en el desarrollo de algunas prácticas más sostenibles. En este enfoque, la moda ya no se puede entender como un fenómeno separado o aislado, sino que está incrustada en prácticas culturales más amplias y donde la sostenibilidad ya no puede ser vista como un 'complemento' o algo separado de ella (de la ropa), sino más bien como algo que surge de las propias prácticas cotidianas vestimentarias (Woodward, 2007). 


\section{Conclusiones}

En primer lugar, en los estudios de la moda hemos podido constatar que, a pesar del prejuicio científico inicial, en los últimos años se han producido numerosas investigaciones dedicadas al estudio de la moda, lo cual nos confirma que se están produciendo un conjunto de conocimientos y actitudes compartidas hacia el tema de la sostenibilidad. En este nuevo campo de estudio, que se viene afianzando, la sostenibilidad se ha constituido en una nueva categoría esencial para el estudio de la moda en el siglo XXI (Mora et al., 2014b).

En segundo lugar, en lo que respecta al universo significativo de la moda sostenible, cabe subrayar su naturaleza interdisciplinar, lo cual significa abarcar un amplio campo de investigación donde es necesario emplear y combinar diversas disciplinas desde la economía, la sociología, la antropología, el derecho y la biología. Y utilizar diversas metodologías: las narrativas analíticas, los estudios estadísticos empíricos, la modelización teórica, el análisis cualitativo o etnográfico (Kawamura, 2011; Mora et al., 2014b).

En tercer lugar, este trabajo también ha querido desafiar el pensamiento convencional de los estudios de moda, a través de los estudios de ciencia y tecnología (CTS) y su método asociado -la teoría del Actor Red (ANT)-, al plantear la investigación de la moda como un "sujeto hibrido entre la naturaleza y la cultura". El entender la moda como un "sujeto híbrido", nos da la oportunidad de ver las continuidades entre las prácticas de vestir y su amplio impacto ambiental. Al mismo tiempo, nos permite pensar de una forma más radical sobre cómo nos conectamos con la naturaleza y la cultura, y, de esta nueva forma, tratar de asignar y ensamblar plenamente las relaciones entre nuestra ropa y el medio ambiente (Latour, 2007; Entwistle, 2015; 2016).

En cuarto lugar, hemos sugerido que la teoría (TPS) nos puede ofrecer formas innovadoras de pensar la moda y la sostenibilidad, aunque el potencial explicativo de estos nuevos enfoques todavía no se ha desarrollado plenamente. Sin embargo, Klepp y Bjerck (2014); Bellotti y Mora (2014); y Woodward (2007) han hecho algunos esfuerzos en este sentido, centrando sus trabajos en el material físico -en lugar del simbólico- $y$, de esta forma, destacando la importancia de la materialidad de la ropa en nuestras relaciones con los objetos/cosas. Lo cual nos permite pensar en la moda como una práctica material y no solo como un mero discurso estético o de desigualdad social.

En quinto lugar, Carfagna et al. (2014); Tseëlon (2011); Crane (2010); Lewis (2013), desde diferentes perspectivas disciplinarias, coinciden en señalar que la mayor dificultad de la moda está en la constitución de un nuevo imaginario de la moda más inclusivo, plural y sostenible. De ahí la siguiente cuestión: ¿cómo pueden las prácticas de consumo, producción y representación alimentar un nuevo imaginario?

\section{Referencias bibliográficas}

Baudrillard, J. (1974). La sociedad de consumo: sus mitos, sus estructuras. Barcelona, España: Plaza \& Janés.

Bellotti, E., y Mora, E. (2014). Networks of practices in critical consumption. Journal of Consumer Culture, 16(3), 718-760. doi: 10.1177/1469540514536191

Black, S. (2008). Eco-Chic: The Fashion Paradox. Londres, Reino Unido: Black Dog Publishing.

Black, S., Entwistle, E., Rocamora, A., De la Haya, A., Root, R., y Thomas, H. (2014). The Handbook of Fashion Studies. Londres, Reino Unido: Bloomsbury Publishing Plc.

Bourdieu, P. (1991). La distinción. Madrid, España: Taurus.

Bovone, L. (2015). Cultura materiale e nuovi valori: il caso della moda ética. Sociología della comunicación, (50), 100-113. doi: 10.3280/SC2015-050010 
Bovone, L., y Mora, E. (2007). La Spesa Responsabile, il consumo biológico e solidable. Roma, Italia: Dozelli editore.

Bradley, H., y Healy, G. (2008). Ethnicity and Gender at Work: Inequalities, Careers and Employment Relations. Nueva York, Estados Unidos: Palgrave Macmillan. doi: 10.1057/9780230582101

Breward, C. (2003). Moda. Oxford, Reino Unido: Oxford University Press.

Callon, M. (1986). Some elements of a sociology of translation: Domestication of the scallops and the fishermen of St Brieuc Bay. En J. Law (Ed.), Power, Action, and Belief: A New Sociology of Knowledge? (pp. 169-233). Londres, Reino Unido: Routledge. doi: 10.1111/j.1467-954X.1984.tbooll3.x

Caniato, F., Caridi, M., Crippa, L., y Moretto, A. (2012). Environmental sustainability in fashion supply chains: An exploratory case based research. International Journal of Production Economics, 135(2), 659-670. doi: 10.1016/j.ijpe.2011.06.001

Canina, M., y Volonté, P. (Eds.). (2014). Overfashion: Nuove prospettive per la moda nella società che ingrassa. Milán, Italia: Franco Angeli.

Carfagna, L., Dubois, E., Fitzmaurice, C., Ouimette, M., Schor, J., Willis, M., y Laidley, T. (2014). An emerging eco-habitus: The reconfiguration of high cultural capital practices among ethical consumers. Journal of Consumer Culture, 14(2), 158-78. doi: 10.1177/1469540514526227

Castells, M. (2000). La empresa en red: cultura, instituciones y organizaciones de la economía informacional. En La Era de la Información: Economía, sociedad y cultura (pp. 179-223). Madrid, España: Alianza.

Choi, T. M. (2013). Local sourcing and fashion quick response system: The impacts of carbon footprint tax. Transportation Research Part E: Logistics and Transportation Review, (55), 43-54. doi: 10.1016/j. tre.2013.03.006

Clark, H. (2008). Slow fashion: an oxymoron or a promise for the future? Fashion Theory: The Journal of Dress, Body \& Culture, 12(4), 427-446. doi: 10.2752/175174108X346922

Cline, E. (2014). La moda desechable. El escandaloso costo de la moda rápida. Barcelona, España: Paidos.

Comisión Europea. (2013). Flash Eurobarometro 367: Attitudes of Europeans towards building the single market for green products. Recuperado de http://ec.europa.eu/public_opinion/archives/ flash_arch_374_361_en.htm\#367

Crane, D. (2000). Fashion and Its Social Agendas: Class, Gender and Identity in Clothing. Chicago, Estados Unidos: University of Chicago Press.

Crane, D. (2010). Environmental change and the future of consumption: implications for consumer identity. Anuario Filosófico, 43(98), 353-379.

Crewe, L. (2004). A thread lost in an endless labyrinth: unravelling fashion's commodity chains. En A. Hughes y S. Reimer (Eds.), Geographies of Commodity Chains (pp. 195-214). Londres, Reino Unido: Routledge.

Doppelt, B. (2012). The Power of Sustainable Thinking. Londres, Reino Unido: Routledge. doi: $10.4324 / 9781849773232$ 
Elkington, J. (1998). Accounting for the triple bottom line. Measuring Business Excellence, 2(3), 18-22. doi: 10.1108/eb025539

Entwistle, J. (2000). The Fashioned Body. Cambridge, Reino Unido: Polity Press.

Entwistle, J. (2015). Sustainability and fashion. En K. Fletcher y M. Tham (Eds.), Routledge Handbook of Sustainability and Fashion (pp. 25-33). Nueva York, Estados Unidos: Bloomsbury. doi: 10.4324/9780203519943.ch2

Entwistle, J. (2016). Bruno Latour: Actor-Network Theory and Fashion. En A. Rocamora y A. Smelik (Eds.), Thinking Through Fashion (pp. 269-285). Londres, Reino Unido: I. B. Tauris \& Co. Ltd.

Entwistle, J., y Slater, D. (2014). Reassembling the Cultural: fashion models, brands and the meaning of 'culture' after ANT. Journal of Cultural Economy, 7(2), 161-177. doi: 10.1080/17530350.2013.783501

Evans, D. (2012). Beyond the throwaway society: ordinary domestic practice and a sociological approach to household food waste. Sociology, 46(1), 41-56. doi: 10.1177\%2F0038038511416150

Fletcher, K. (2008). Sustainable Fashion and Textiles. Design Journey. Londres, Reino Unido: Earthscan.

Fletcher, K. (2010). Slow fashion: an invitation for systems change. Fashion Practice: The Journal of Design, Creative Process and the Fashion, 2(2), 259-266. doi: 10.2752/175693810X1277462538759

Fletcher, K., y Grose, L. (2012). Fashion \& Sustainability: Design for Change. Londres, Reino Unido: Laurence King Publishing.

Gardetti, M. (2018). Textiles y moda. ¿Qué es la sostenibilidad? Madrid, España: Lid editorial.

Gardetti, M., y Delgado, M. (2018). Vestir un mundo sostenible, Madrid, España: Lid editorial.

Gereffi, G., y Korzeniewicz, M. (1994). Commodity Chains and Global Capitalism. Westport, Estados Unidos: Greenwood Press.

Gwilt, A. (2014). A Practical Guide to Sustainable Fashion. Nueva York, Estados Unidos: The Bloomsbury Group. doi: 10.5040/9781474218559

Gwilt, A., y Rissanen, T. (2011). Shaping Sustainable Fashion. Changing the Way we Make and Use Clothes. Londres, Reino Unido: Earthscan.

Hansen, K. T. (2000). Salaula: The World of Secondhand Clothing and Zambia. Chicago, Estados Unidos: University of Chicago Press.

Harris, B. (2017). Famine and Fashion: Needlewomen in the Nineteenth Century. Londres, Reino Unido: Routledge. doi: $10.4324 / 9781315255446$

Heike, J. (2015). Fashioning Memory: Vintage Style and Youth Culture. Londres, Reino Unido: Bloomsbury Academic.

Henninger, C., Alevizou, P., y Oates, C. (2016). What is sustainable fashion. Journal of Fashion Marketing and Management: An International Journal, 20(4), 400-416. doi: 10.1108/JFMM-07-2015-0052

Hethorn, J., y Ulasewicz, C. (2008). Sustainable Fashion, Why Now? A Conversation about Issues, Practices, and Possibilities. Nueva York, Estados Unidos: Fairchild Books. 
Jackson, T. (2011). Prosperity without growth: Economics for a Finite Planet. Londres, Reino Unido: Routledge. doi: 10.1260/0958-305X.22.7.1013

Jucker, L. (2012). The labyrinth of labels and certificates. En M. Ricchetti y M. Frisa (Eds.), The Beautiful and the Good: Reasons for Sustainable Fashion (pp. 147-160). Venecia, Italia: Marsilio.

Kawamura, Y. (2005). Fashion-ology: An Introduction to Fashion Studies. Oxford, Reino Unido: Berg. doi: $10.2752 / 9781847888730$

Kawamura, Y. (2011). Doing Research in Fashion and Dress: An Introduction to Qualitative Methods. Nueva York, Estados Unidos: Berg.

Klein, N. (2001). No Logo. Buenos Aires, Argentina: Paidos.

Klepp, I. G., y Bjerck, M. (2014). A methodological approach to the materiality of clothing: Wardrobe studies. International Journal of Social Research Methodology, 17(4), 373-386. doi: $10.1080 / 13645579.2012 .737148$

Latour, B (2007). Nunca fuimos modernos, Ensayo de antropología simétrica. Buenos Aires, Argentina: Siglo XXI.

Latour, B. (2008). Reensamblar lo social. Una teoría a la introducción del actor red. Buenos Aires, Argentina: Manantial.

Latour, B., y Woolgar, S. (1995). La vida en el laboratorio. La construcción de los hechos científicos. Madrid, España: Alianza.

Lewis, R. (2013). Modest Fashion: Styling Bodies, Mediating Faith. Londres, Reino Unido: I.B. Tauris.

Lipovetsky, G. (1990). El imperio de lo efímero. La moda y su destino en las sociedades modernas. Barcelona, España: Anagrama.

Lohmeyer, N., y Schüßler, E. (2017). Rana Plaza as a threat to the fast fashion model? An analysis of institutional responses to the disaster in Germany. En C. Becker-Leifhold y M. Heuer (Eds.), Eco Friendly and Fair: Fast Fashion and Consumer Behavior (pp. 3-14). Londres/Nueva York, Reino Unido/Estados Unidos: Routledge. doi: 10.4324/9781351058353-1

Lunghi, C. (2012). Creative evasioni: Manifatture di moda in carcere. Milán, Italia: Franco Angeli.

Lunghi, C., y Montagnini, E. (2007). La moda della responsabilità. Milán, Italia: Franco Angeli.

Lury, C. (2011). Consumer culture. Cambridge, Reino Unido: Polity Press.

MacVaugh, J., y Norton, M. (2012). Introducing sustainability into business education contexts using active learning. International Journal of Sustainability in Higher Education, 13(1), 72-8. doi: $10.1108 / 14676371211190326$

Maffesoli, M. (1990). El tiempo de las tribus. Barcelona, España: Icaria.

Martínez-Barreiro, A. (1998). La moda en las sociedades modernas. Madrid, España: Técnos.

McDonough, W., y Braungart, M. (2002). Cradle to Cradle: Remaking the Way We Make Things. Nueva York, Estados Unidos: North Point Press. 
Menkes, S. (2012). Bags Made by Hand, in East Africa. New York Times. Recuperado de https://www. nytimes.com/2012/09/04/fashion/bags-made-by-hand-in-east-africa.html

Mora, E., Rocamora, A., y Volonté, P. (2014a). The internationalization of fashion studies: Rethinking the peer-reviewing process. International Journal of Fashion Studies, 1(1), 3-17. doi: 10.1386/infs.1.1.3_2

Mora, E., Rocamora A., y Volonté, P. (2014b). On the issue of sustainability in fashion studies. International Journal of fashion Studies, 11(2), 139-147. doi: 10.1386/infs.1.2.139_1

Pantzar, M., y Shove, E. (2005). Understanding innovation in practice. A discussion of the production and reproduction of Nordic Walking. Technology Analysis and Strategic Management, 22(4), 447462. doi: 10.1080/09537321003714402

Parker, L., y Dickson, M. (2009). Sustainable fashion: A handbook for educators. Bristol, Reino Unido: Labour Behind the Label.

Paulicelli, E., y Wissinger, E. (2013). Introduction. WSQ: Women's Studies Quarterly, 41(1), 14-27. doi: $10.1353 /$ wsq.2013.0065

Pedersen, E., y Gwodzd, W. (2014). From resistance to opportunity seeking: Strategic responses to institutional pressures for corporate social responsibility in the Nordic fashion industry. Journal of Business Ethics, 119(2) 245-64. doi: 10.1007/s10551-013-1630-5

Phizacklea, A. (1990). Unpacking the Fashion Industry: Gender, Racism and Class in Production. Londres, Reino Unido: Routledge.

Raghuram, P. (2004). Initiating the commodity chain: South Asian women and fashion in the diaspora. En A. Hughes y S. Reimer (Eds.), Geographies of Commodity Chains (pp. 120-136). Londres, Reino Unido: Routledge.

Rinaldi, F., y Testa, S. (2014). The Responsible Fashion Company. Londres, Reino Unido: Routledge.

Rocamora, A. y Smelik, A. (Eds.). (2016). Thinking Through Fashion. Londres, Reino Unido: I. B. Tauris.

Ross, A. (1997). No Sweat: Fashion, Free Trade and the Rights of Garment Workers. New York, Estados Unidos: Verso.

Salcedo, E. (2014). Moda ética para un futuro sostenible. Madrid, España: Gustavo Gili.

Santagata, W. (2010). The Culture Factory: Creativity and the Production of Culture. Berlín, Alemania: Springer. doi: 10.1007/978-3-642-13358-9

Schatzki, T. (1996). Social practices: a wittgensteinian approach to human activity and the social. Nueva York, Estados Unidos: Cambridge University Press.

Seidman, D. (2007). How, Why How. We Do Anything Means Everything. Nueva Jersey, Estados Unidos: John Wiley \& Sons.

Sennett, R. (2008). The Craftsman. New Haven, Estados Unidos: Yale University Press.

Shen, B. (2014). Sustainable Fashion Supply Chain: Lessons from H\&M. Sustainability, 6(9), 6236-6249. doi: $10.3390 /$ su6096236 
Shove, E. (2003). Comfort, Cleanliness and Convenience: The Social Organization of Normality. Oxford, Reino Unido: Berg.

Shove, E. (2010). Beyond the ABC: climate change policy and theories of social change. Environment and Planning, (42), 1273-1285. doi: 10.1068/a42282

Shove, E., y Pantzar, M. (2005). Consumers, producers and practices: understanding the invention and reinvention of Nordic walking. Journal of ConsumerCulture, 5(1),43-64. doi:10.1177/1469540505049846

Shove, E., Pantzar, M., y Watson, M. (2012). The Dynamics of Social Practice: Everyday Life and How it changes. Londres, Reino Unido: Sage.

Siegle, L. (2011). To Die For: Is Fashion Wearing Out the World? Londres, Reino Unido: Fourth Estate.

Simmel, G. (1957). Fashion. American Journal of Sociology, 62(6), 541-558.

Skjerven, A., y Reitan, J. (2017). Design for a Sustainable Culture: Perspectives, Practices and Education. Londres, Reino Unido: Routledge.

Slater, K. (2003). Environmental Impact of Textiles: Production. Processes and Protection. Cambridge, Reino Unido: Woodhead Publishing Limited/The Textile Institute.

Suzuki, D., y Dressler, H. (2002). Good News for a Change: How Everyday People are Helping the Planet. Toronto, Canada: Stoddart Publishing Co.

Tseëlon, E. (1995). The Masque of Femininity. Londres, Reino Unido: Sage.

Tseëlon, E. (2011). Introduction: A Critique of the Ethical Fashion Paradigm. Critical Studies in Fashion and Beauty, 2(1), 3-68. doi: 10.1386/csfb.2.1-2.3_2

UNESCO -Organización de las Naciones Unidas para la Educación, la Ciencia y la Cultura-. (2005). El Decenio de las Naciones Unidas de la Educación para el Desarrollo Sostenible (EDS) (2005-2014). Recuperado de https://es.unesco.org/themes/educacion-desarrollo-sostenible/comprender-EDS/ decenio-onu

Van der Velden, N. M., Patel, M. K., y Vogtländer, J. G. (2014). LCA benchmarking study on textiles made of cotton, polyester, nylon, acryl, or elastane. The International Journal of Life Cycle Assessment, 19(2), 331-356. doi: 10.1007/s11367-013-0626-9

Veblen, T. (1974). La teoría de la clase ociosa. Ciudad de México, México: Fondo de Cultura Económica.

Wallerstein, I. (1974). The modern world system. Berkeley, Estados Unidos: University of California Press.

Warde, A. (2005). Consumption and the theory of practice. Journal of Consumer Culture, 5(2), 131-154. doi: $10.1177 / 1469540505053090$

Warde, A., y Southerton, D. (2012). Introduction. Preamble: social sciences and sustainable consumption. En A. Warde y D. Southerton (Eds.), The Habits of Consumption (pp. 1-25). Helsinki, Finlandia: Helsinki Collegium for Advanced Studies.

Warde, A., Cheng, S. Olsen, W., y Southerton, D. (2007). Changes in the practice of eating. A comparative analysis of time-use. Acta Sociologica, 50(4), 363-385. doi: 10.1177/ 0001699307083978 
Ana Martínez-Barreiro

WCED -World Commission on Environment and Development-. (1987). Our Common Future (The Brundtland Report). Oxford, Reino Unido: Oxford University Press.

Welters, L., y Lillethun, A. (2011). The Fashion Reader. 2nd edition. Oxford, Reino Unido: Berg.

Woodward, S. (2007). Why Women Wear What They Wear. Oxford, Reino Unido: Berg. 